\title{
MULTIPLE MONONEURITIS ASSOCIATED WITH ISCHEMIC BRAIN LESIONS IN SYSTEMICLUPUSERYTHEMATOSUS AND TRIPLE POSITIVE ANTIPHOSPHOLIPID ANTIBODIES
}

Victor Matheus Ostrovski Souza Santos ${ }^{1, \star}$, Jean Michell Correia Monteiro ${ }^{1}$, Cipriano Reis Gama ${ }^{1}$, Pablo Vinícius da Fonseca ${ }^{1}$, Henrique Ayres Mayrink Giardini ${ }^{1}$, Rosa Maria Rodrigues Pereira ${ }^{1}$

1.Universidade de São Paulo, São Paulo (SP), Brazil.

Corresponding author: victormatheusoss1@gmail.com

\section{BACKGROUND}

Neurological manifestations of systemic lupus erythematosus (SLE) are usually challenging, but when clinical syndromes with focal deficits manifest quickly, adequate investigation and treatment are critical to prevent irreversible complications. We report a case of involvement of the peripheral and central nervous system in a patient with recent SLE diagnostic associated with antiphospholipid antibodies.

\section{CASE REPORT}

A 36-year-old woman, without previous diseases, diagnosed with cutaneous and articular SLE manifestation, ANA homogeneous pattern 1/320 (negative anti-DNAds and anti-Sm) and consumption of complement, for six months, was referred for rheumatology investigation because she had burning pain in her lower limbs for four months, which evolved with progressive distal weakness and inability to walk. At the initial assessment, the patient had significant bilateral distal weakness. For etiological investigation, neuroaxis magnetic resonance was performed, which demonstrated the presence of several foci of hypersignal in T2 / FLAIR in the white matter of the cerebral hemispheres and areas with hypersignal in FLAIR with restriction to diffusion in the nuclei of the base, suggestive of ischemia, and electroneuromyography of the lower limbs, suggestive of multiple mononeuritis (Figs. 1 and 2). There was no change in cerebrospinal fluid. Anticardiolipin antibodies, anti-beta 2 glycoprotein I antibodies and lupus anticoagulant were positive. Due to the severity of the case, pulse therapy with methylprednisolone and cyclophosphamide were indicated and full anticoagulation was started.
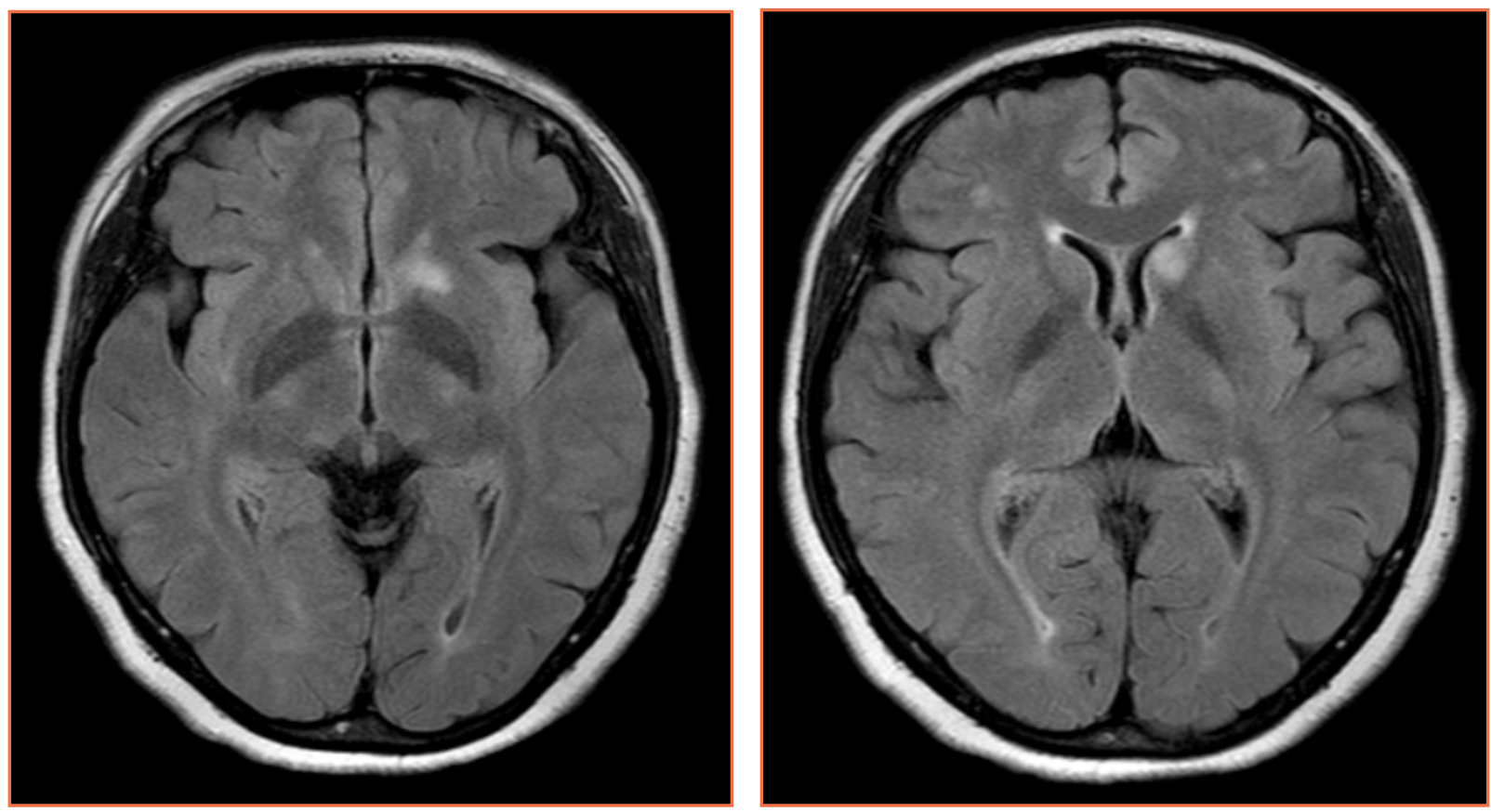

Figure 1. Diffusion restriction areas in the left anterior nucleocapsular region, affecting the head of the caudate nucleus, the anterior branch of the internal capsule and the anterior aspect of the putamen, of subacute ischemic nature (FLAIR). 


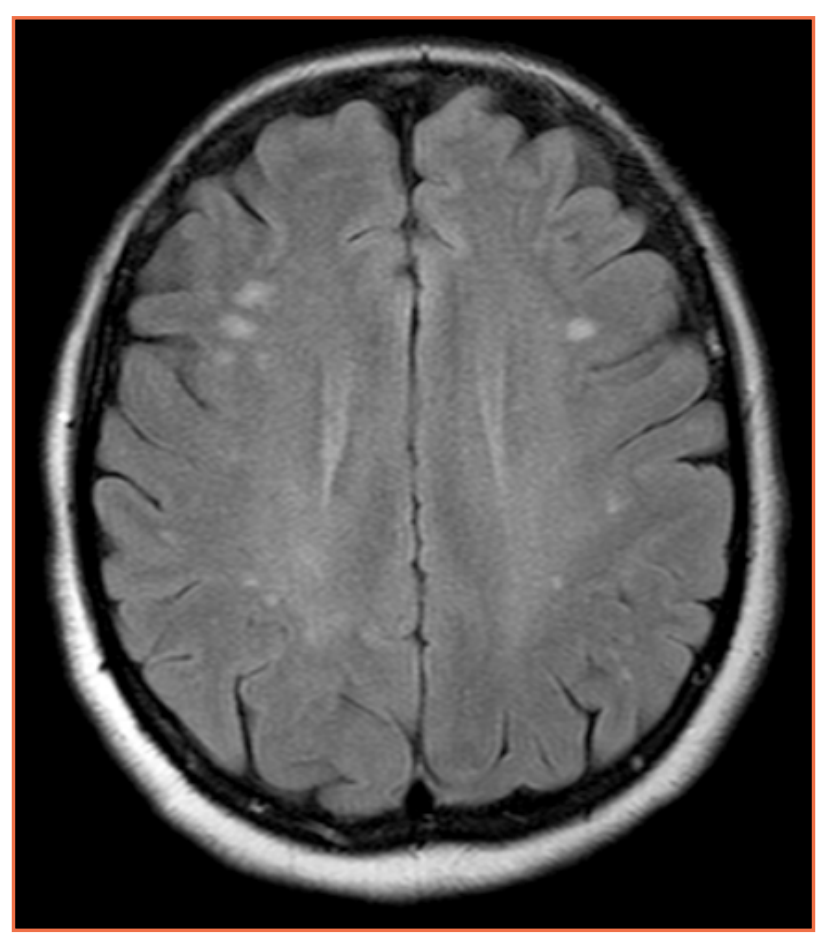

Figure 2. Hypersignal foci in FLAIR in the white matter of the cerebral hemispheres, sparse in the subcortical regions - suggestive of myelinated rarefaction/gliosis in the territory of small vessels.

\section{CONCLUSION}

Approximately 10 to $15 \%$ of SLE patients develop peripheral neuropathy probably due to vasculitis of small arteries that irrigate the affected nerves, with polyneuropathy and multiple mononeuritis being the most common presentations. In addition, antiphospholipid antibodies play an important role in the ischemic pathway that leads to thrombosis and complement activation, with a well-documented relationship between neuropsychiatric manifestations and elevated serum levels of anticardiolipin antibodies and lupus anticoagulant. Central and peripheral neurological manifestations can occur simultaneously in patients with SLE associated or not with antiphospholipid antibodies and a high degree of suspicion must be maintained for the diagnosis and proper management of these complex cases. 